\title{
Synthesis of 3-Benzylcoumarins Using Suzuki Coupling Reaction
}

\author{
Young Bum Kwon, JinHyung Park, Bo Ram Choi, Hyun Soo Kim, and Cheol Min Yoon* \\ Department of Advanced Material Chemistry, Korea University, Korea ${ }^{*}$ E-mail: cmyoon@korea.ac.kr \\ Received March 5, 2011, Accepted June 28, 2011
}

Key Words : Coumarin, Arylboronic acids, Palladium acetate, Boronic acid, Suzuki coupling

Coumarins are a structural scaffold in the numerous natural product $^{1}$ and one of well known oxygen containing heterocycles showing a variety of biological activities. ${ }^{2}$ In addition, they have found in the technological applications ${ }^{3}$ and used as intermediate for the synthesis of important molecules. ${ }^{4}$ Because of their diverse applications, a variety of classical routes $^{5}$ to coumarins such as Pechmann, Knoevenagel, Perkin, Reformatsky and Wittig condensation reactions have been reported. However, these methods have their own problems such as harsh reaction conditions, multistep synthesis or low chemical yield. To overcome the problems associated with these classical methods, many strategies for the convenient and versatile synthesis of coumarin derivatives by direct ring formations ${ }^{6}$ and by metal catalyzed coupling reactions of coumarin derivatives ${ }^{7,8}$ have recently been developed.

Among coumarin derivatives, 3-alkylcoumarins including benzylcoumarins are important building block and showed important biological activities. ${ }^{9}$ As an example, Warfarin, a 3benzylcoumarin derivative, is the most widely used oral anticoagulant. ${ }^{10} \mathrm{New}$, convenient, and versatile syntheses of 3-alkylcoumarins has been developed. ${ }^{9}$ Wadia et al. reported the synthesis of 3-benzylcoumarins from the reaction of a complex of the amides and $\mathrm{POCl}_{3}$ with substituted salicylaldehydes. ${ }^{9}$ Bräse and his colleagues synthesized successfully the 3-benzylcoumarins from the salicylaldehydes and cinnamyl aldehydes using nucleophilic carbenes in ionic liquid in one-step. ${ }^{9}$ Recently, 3 -allylcoumarin was prepared by decarboxylative allylation of allyl ester of 3-carboxylcoumarin using palladium catalyst under mild condition. ${ }^{9}$ But those methods have several drawbacks such as limited number of substrates, and harsh reaction condition.

Palladium catalyzed cross coupling reactions have emerged as a powerful method for the carbon-carbon bond formation. Among them, Suzuki coupling is the most widely used method, because organoboronic acids are generally non-toxic and thermally, air-, and moisture-stable. The palladium catalyzed benzyl halides and allyl halide coupling with organoboronic acids are also well established ${ }^{11}$ and the reaction has been known to proceed through $\eta^{3}$-complex. ${ }^{12}$ Here, we report the efficient cross coupling reaction of 3-(chloromethyl)coumarins prepared from salicylaldehydes ${ }^{13}$ with arylboronic acid to give the corresponding 3-benzylcoumarins in excellent yields (Scheme 1).

The reaction of 3 -(chloromethyl)coumarin $\mathbf{1 a}$ and

${ }^{\top}$ This paper is dedicated to Professor Eun Lee on the occasion of his honourable retirement. phenylboronic acid as a model was first examined using palladium acetate and $\mathrm{Na}_{2} \mathrm{CO}_{3}$ as a base in methanol.

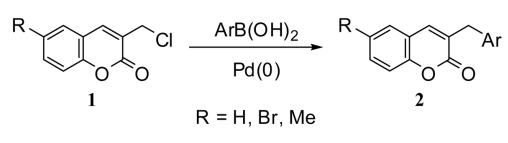

Scheme 1

Table 1. The reactions ${ }^{a}$ of 3-(chlorometyl)columarins with arylboronic acids $^{14}$

Entry

${ }^{a}$ Reaction condition: 3 -(chloromethyl)coumarin, arylboronic acids $(1.2$ equiv), $\mathrm{Pd}(\mathrm{OAc})_{2}\left(0.03\right.$ equiv.), $\mathrm{Na}_{2} \mathrm{CO}_{3}$ (2.0 equiv.) in methanol at $\mathrm{rt}$ for 1 h. ${ }^{b}$ Isolated yield.

Luckily enough, the reaction of 3-(chloromethyl)coumarin 1a with phenyl boronic acid (1.2 equiv.) in the presence of $\mathrm{Na}_{2} \mathrm{CO}_{3}$ (2.0 equiv.) and $\mathrm{Pd}(\mathrm{OAc})_{2}(0.03$ equiv.) was working very nicely in methanol at rt to give a benzylcoumarin in 94 $\%$ isolated yield. With this condition in hand, the coupling reaction of coumarin 1a with 4-substitutedbenzene boronic acids such as 4-methoxy, 4-methyl and 4-chloromethyl coumarins was attempted to afford the corresponding coupling products in excellent respective yields (entries 2-4 


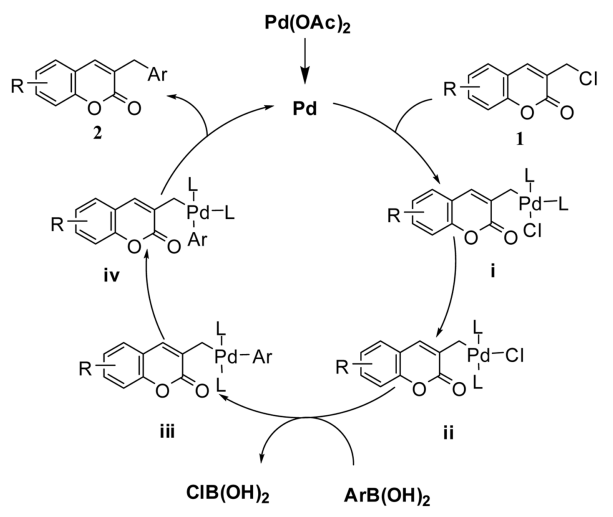

Scheme 2

of Table 1). 6-Bromo-3-(chloromethyl)coumarin $\mathbf{1 b}$ and 3(chloromethyl)-6-methylcoumarin 1c, underwent crosscoupling reaction with four different arylboronic acids nicely under our condition to give the corresponding coupling products 2e-h in excellent yields (entries 5-12 of Table 1). Bromo functional group of bromophenyl of $\mathbf{1 b}$ was remained in intact under the reaction condition. All the reaction is efficient and finished within $1 \mathrm{hr}$.

The reaction might be proceeded through the cis-oxidative addition of 3-(chloromethyl)coumarin 1 to $\mathrm{Pd}(0)$ to give $\mathrm{Pd}$ -complex followed by isomerization to give intermediate ii, which is transmetallated and isomerized to cis-isomer iv followed by cis-reductive elimination to afford the coupling product 2. (Scheme 2)

In conclusion, 3-benzylcoumarins were prepared from the reaction of 3-(chloromethyl)-coumarins with arylboronic acids in the presence of palladium acetate and sodium carbonate in methanol at room temperature in excellent yields. The reaction is very simple, fast, and efficient method to prepare 3-benzylcoumarins.

Acknowledgments. This work was supported by Korea University.

\section{Reference and Notes}

1. Hepworth, J. D.; Gabbutt, C. D.; Heron, B.M. In Comprehensive Heterocyclic Chemistry-II; Katritzky, A. R., Rees, C. W., Scriven, E. F. V., Eds.; Pergamon: New York, 1996; Vol. 5, pp 417-434.

2. (a) Neyts, J.; Clercq, E. D.; Singha, R.; Chang, Y. H.; Das, A. R.; Chakraborty, S. K.; Hong, S. C.; Tsay, S. C.; Hsu, M. H.; Hwu, J. R. J. Med. Chem. 2009, 52, 1486. (b) O'Kennedy, R., Thornes, R. D., Eds.; In Coumarins: Biology, Applications and Mode of Action; Wiley: Chichester, U.K. 1997.

3. (a) Brun, M. P.; Bischoff, L.; Garbay, C. Angew. Chem., Int. Ed. 2004, 43, 3432. (b) Lee, J. H.; Jeong, A. R.; Shin, I-.S.; Kim, HJ.; Hong, J.-I. Organic Lett. 2010, 12, 764.

4. Jung, M. E.; Allen, D. A. Organic Lett. 2009, 11, 757. (b) Chen, G.; Tokunaga, N.; Hayashi, T. Organic Lett. 2005, 7, 2285. (c) Stoffman, E. J. L.; Clive, D. L. K. Org. Biomol. Chem. 2009, 7, 4862.

5. (a) von Pechmann, H. Chem. Ber. 1883, 16, 2119. (b) Rabjohn, N. Org. React. 1976, 24, 261. (c) Sugino, T.; Tanaka, K. Chemistry Lett. 2001, 513. (d) Jones, G. Org. React. 1967, 15, 204. (e) Brufola, G.; Fringuelli, F.; Piermatti, O.; Pizzo, F. Heterocycle
1996, 43, 1257. (f) Perkin, W. H. J. Chem. Soc. 1875, 28, 11. (g) Johnson, J. R. Org. React. 1942, 1, 210. (h) Shriner, R. L. Org. React. 1942, 1, 1. (i) Narasimhan, N. S.; Mali, R. S.; Barve, M. V. Synthesis 1979, 906. (j) Yavari, I.; Hekmat-Shoar, R.; Zonouzi, A. Tetrahedron Lett. 1998, 39, 2391.

6. (a) Ramesh, E.; Raghunathan, R. Tetrahedron Lett. 2008, 49, 1812. (b) Upadhyay, P. K.; Kumar, P. Tetrahedron Lett. 2009, 50, 236.

7. (a) Wang, W.; Ding, Q.; Fana, R.; Wua, J.Tetrahedron Lett. 2007, 48, 3647. (b) Das, A. R.; Medda, A.; Singha, R. Tetrahedron Lett. 2010, $51,1099$.

8. (a) Wu, J.; Wang, L.; Fathi, R.; Yang, Z. Tetrahedron Lett. 2002, 43, 4395. (b) Wu, J.; Zhanga, L.; Xiaa, H.-G. Tetrahedron Lett. 2006, 47, 1525. (c) Zhang, L.; Meng, T.; Fan, R.; Wu, J. J. Org. Chem. 2007, 72, 7279.

9. (a) Torang, J.; Vanderheiden, S.; Nieger, M.; Bräse, S. Eur. J. Org. Chem. 2007, 943 and references cited therein. (b) Britto, N.; Gore, V. G.; Mali, R. S.; Ranade, A. C. Synth. Commun. 1989, 19, 1899. (c) Jana, R.; Trivedi, R.; Tunge, J. A. Organic Lett. 2009, 11, 3434.

10. Johnson, J. A. Pharmacotherapy 2008, 28, 1081.

11. (a) Nichele, T. Z.; Monteiro, A. L. Tetrahedron Lett. 2007, 48, 7472. (b) Kuwano, R.; Yokogi, M. Chem. Commun. 2005, 5899.

12. (a) Roberts, J. S.; Klabunde, K. J. J. Am. Chem. Soc. 1977, 99, 2509. (b) Kuwano, R.; Kondo, Y.; Matsuyama, Y. J. Am. Chem. Soc. 2003, 125, 12104.

13. (a) Kaye, P. T.; Musa, M. A.; Nocanda, X. W. Synthesis 2003, 531. (b) Kaye, P. T.; Musa, M. A. Synth. Commun. 2004, 34, 3409.

14. A typical procedure of synthesis of 3-benzylcoumarin (2a): ${ }^{9 \mathrm{a}}$ To a solution of 3-(chloromethyl)coumarin 1a $(100 \mathrm{mg}, 0.51 \mathrm{mmol})$ and $\mathrm{Na}_{2} \mathrm{CO}_{3}$ (109 mg, 2.0 equiv.) in methanol $(3 \mathrm{ml})$ in a reaction vessel was added benzene boronic acid $(75 \mathrm{mg}, 1.2$ equiv.) and $\mathrm{Pd}(\mathrm{OAc})_{2}(3.5 \mathrm{mg}, 0.03$ equiv.) under argon atmosphere. The resulting mixture was stirred for $1 \mathrm{~h}$ at $\mathrm{rt}$. And then the reaction was filtered through celite pad, concentrated, and purified by silica-gel chromatography using an eluent solution of ethyl acetate and hexane $(1: 10, \mathrm{v} / \mathrm{v})$ to give a 3-benzylcoumarin 2a $(912 \mathrm{mg}$, $91.9 \%)$ as a white solid. ${ }^{1} \mathrm{H}$ NMR $\left(\mathrm{CDCl}_{3}, 300 \mathrm{MHz}\right) \delta$ 7.49-7.43 $(\mathrm{t}, J=7.8 \mathrm{~Hz}, 1 \mathrm{H}), 7.39-7.20(\mathrm{~m}, 9 \mathrm{H}), 3.90(\mathrm{~s}, 2 \mathrm{H}) .{ }^{13} \mathrm{C} \mathrm{NMR}$ $\left(\mathrm{CDCl}_{3}, 75 \mathrm{MHz}\right) \delta 161.70,153.17,139.38,137.74,130.91$, $129.49,129.42,128.89,127.50,126.96,124.40,119.52,116.65$, 36.66. IR (neat) : $\tilde{v} 1713 \mathrm{~cm}^{-1}$ (s).

15. Data of Representative coupling products:

3-(4-Methylbenzyl) coumarin (2c): White solid. mp: $107-108{ }^{\circ} \mathrm{C}$. ${ }^{1} \mathrm{H}$ NMR $\left(\mathrm{CDCl}_{3}, 300 \mathrm{MHz}\right) \delta$ 7.48-7.43 (m, 2H), 7.37-7.14 (m, $7 \mathrm{H}), 3.86(\mathrm{~s}, 2 \mathrm{H}), 2.36(\mathrm{~s}, 3 \mathrm{H}) .{ }^{13} \mathrm{C} \mathrm{NMR}\left(\mathrm{CDCl}_{3}, 75 \mathrm{MHz}\right) \delta$ $161.71,153.02,139.09,136.39,134.49,130.69,129.63,129.44$, $129.27,127.34,124.22,119.45,116.37,36.11,21.08$. MS (EI) $\mathrm{m} /$ $z: 250\left(\mathrm{M}^{+}\right)$. IR (neat): $\tilde{v} 1701 \mathrm{~cm}^{-1}(\mathrm{~s})$.

3-Benzyl-6-bromocoumarin (2e): White solid. $\mathrm{mp}: 138-140{ }^{\circ} \mathrm{C}$. ${ }^{1} \mathrm{H}$ NMR $\left(\mathrm{CDCl}_{3}, 300 \mathrm{MHz}\right) \delta 7.52(\mathrm{td}, J=9.0 \mathrm{~Hz}, 2.1 \mathrm{~Hz}, 2 \mathrm{H})$, 7.39-7.16 (m, 7H), $3.89(\mathrm{~s}, 2 \mathrm{H}) .{ }^{13} \mathrm{C} \mathrm{NMR}\left(\mathrm{CDCl}_{3}, 75 \mathrm{MHz}\right) \delta$ $160.99,151.88,137.83,137.13,133.51,130.86,129.72,129.42$, $128.88,127.03,120.96,118.15,116.83,36.60$. MS (EI) $m / z: 314$ $\left(\mathrm{M}^{+}\right)$. IR (neat): $\tilde{v} 1716 \mathrm{~cm}^{-1}(\mathrm{~s})$.

6-Bromo-3-(4-methoxybenzyl)coumarin (2f): White solid. $\mathrm{mp}$ : $115-116{ }^{\circ} \mathrm{C} .{ }^{1} \mathrm{H}$ NMR $\left(\mathrm{CDCl}_{3}, 300 \mathrm{MHz}\right) \delta 7.53(\mathrm{dd}, J=8.7 \mathrm{~Hz}$, $2.1 \mathrm{~Hz}, 1 \mathrm{H}), 7.49(\mathrm{sd}, J=2.1 \mathrm{~Hz}, 1 \mathrm{H}), 7.20-7.14(\mathrm{~m}, 4 \mathrm{H}) 6.89(\mathrm{~d}$, $J=8.7 \mathrm{~Hz}, 2 \mathrm{H}) 3.81(\mathrm{~s}, 2 \mathrm{H}+3 \mathrm{H}) \cdot{ }^{13} \mathrm{C} \mathrm{NMR}\left(\mathrm{CDCl}_{3}, 75 \mathrm{MHz}\right) \delta$ $161.04,158.62,151.85,137.61,133.43,131.27,130.46,129.69$, $129.00,121.01,118.13,116.80,114.28,55.30,35.76$. MS (EI) $\mathrm{m} /$ $z: 344\left(\mathrm{M}^{+}\right)$. IR (neat): $\tilde{v} 1716 \mathrm{~cm}^{-1}(\mathrm{~s})$.

3-(4-Chlorobenzyl)-6-methylcoumarin (2l): White solid. mp: 120$121^{\circ} \mathrm{C} .{ }^{1} \mathrm{H}$ NMR $\left(\mathrm{CDCl}_{3}, 300 \mathrm{MHz}\right) \delta$ 7.32-7.17 (m, 8H) $3.84(\mathrm{~s}$, $2 \mathrm{H}) 2.36(\mathrm{~s}, 3 \mathrm{H}) .{ }^{13} \mathrm{C} \mathrm{NMR}\left(\mathrm{CDCl}_{3}, 75 \mathrm{MHz}\right) \delta 161.76,151.26$, $139.42,136.28,134.05,132.68,131.98,130.69,128.87,128.69$, 127.28, 119.03, 116.19, 36.04, 20.74. MS (EI) $\mathrm{m} / z: 284\left(\mathrm{M}^{+}\right)$. IR (neat): $\tilde{v} 1709 \mathrm{~cm}^{-1}(\mathrm{~s})$. 